\title{
Cross-correlations mediated by Majorana bound states
}

\author{
Peiyue Wang, ${ }^{1}$ Yunshan Cao, ${ }^{2,1}$ Ming Gong, ${ }^{3}$ 用 Gang Xiong, ${ }^{1}$ and Xin-Qi Li ${ }^{1}$ 用 \\ ${ }^{1}$ Department of Physics, Beijing Normal University, Beijing 100875, China \\ ${ }^{2}$ School of Physics, Peking University, Beijing 100871, China \\ ${ }^{3}$ Department of Physics, The University of Texas at Dallas, Richardson, Texas, 75080 USA
}

\begin{abstract}
We consider the parallel transport through two quantum dots correlated by a semiconductor nanowire which may support a pair of Majorana bound states at the ends. In addition to transient dynamics, via modulating the quantum dot levels, we reveal a characteristic feature of symmetry and antisymmetry in the spectral density of cross correlation mediated by Majorana fermion. We also find an intriguing behavior of vanished cross correlation when one of the dot levels is in resonance with the Majorana zero mode.
\end{abstract}

PACS numbers: 71.10.Pm,74.78.Na,74.45.+c

It has attracted considerable attention in the past years to search for Majorana fermion [1], 2] in solid states, such as the $5 / 2$ fractional quantum Hall system[3] and the $p$ wave superconductor and superfluid[4, 5]. In particular, it was predicted that a semiconductor nanowire, with strong spin-orbit coupling and subject to external magnetic field, may support zero-energy Majorana bound state (MBS) when the nanowire is in proximity to an $s$-wave superconductor[6]. 8 . This proposal has the major advantage of requiring only the most conventional materials, being thus easier to implement in experiments. Indeed, in some recent experiments[12], evidences of the MBSs were spotted in this sort of hybrid nanowire systems.

The realization and manipulation of Majorana fermion in solid-states may pave a way for the desirable faulttolerant topological quantum computation $13-16]$. In pure physics, the Majorana study in solid states is intriguing because of many unusual effects, such as the nonAbelian statistics [? ], the sharp jump in conductance peak [17], the peculiar noise behaviors [18 22], and the $4 \pi$ periodic Majorana-Josephson currents [4, 6, 23, 24, etc. Remarkably, most of these effects are associated with the nonlocal nature of the Majorana fermion,25,27]. The novel nonlocality feature can be most surprisingly elaborated by the example of its nanowire realization. In this case, the emerged pair of MBSs at the ends of the nanowire constitutes, respectively, the real and imaginary parts of an ordinary fermion. This means that, if an electron with energy smaller than the energy gap between the Majorana zero mode and other exited states is injected into the nanowire, the electron would split into two Majorana bound states which are essentially correlated but spatially separated. In some sense the Majorana's nonlocality can be related to a nonlocal cross Andreev reflection (CAR) process [19, 25], because of the presence of superconductivity. However, as described above, the concept of nonlocality of Majorana fermion itself has richer information and is more intriguing than the CAR phenomenon. In Ref. [28] the nonlocal CAR process has been exploited for quantum teleportation, following the same idea of the pio-

\footnotetext{
*Electronic address: kkvlark.gong@gmail.com

†Electronic address: lixinqi@bnu.edu.cn
}

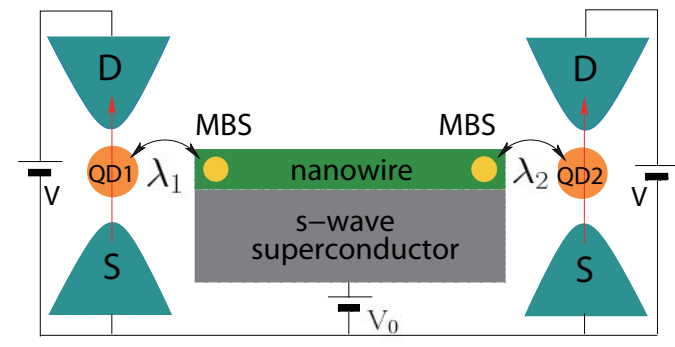

FIG. 1: (Color online) Schematic setup for a cross-correlated parallel transport through two quantum dots which are weakly tunnel-coupled by a semiconductor nanowire. The nanowire is in contact with an $s$-wave superconductor. Under appropriate conditions, such as with large Zeeman splitting and strong spinorbit interaction, a pair of Majorana bound states is anticipated to emerge at the ends of the nanowire. Here we explicitly display the closed circuit that satisfies the law of current conservation.

neering work by Bennett et al. 29]. In such teleportation scheme, what is teleportated is the "state information", but not a "matter" as possibly mediated by the Majorana fermion, based on its nonlocality nature [26, 27, 30].

In this work, we attempt to study the Majorana's nonlocality in a rather direct way. As schematically shown in Fig. 1, we consider a setup of parallel transports through two quantum dots (QDs) coupled by a semiconductor nanowire which may support a pair of MBSs at the ends. We will focus on the cross correlation of currents mediated by the MBSs and reveal some unusual behaviors.

Model and Method.- The transport setup is schematically shown in Fig. 1, where the semiconductor nanowire is in proximity to a conventional $s$-wave superconductor and subject to a Zeeman field. When the Zeeman splitting energy $V_{z}$, the proximity-induced order parameter $\Delta$, and the chemical potential $\mu$ satisfy the condition $V_{z}>\sqrt{\Delta^{2}+\mu^{2}}$, the nanowire is driven into a topological superconducting phase and a pair of zero-energy MBSs will emerge at the ends of the nanowire [6/8]. As a result, the two quantum dots which are tunnel-coupled to the ends of the nanowire are now effectively coupled to the MBSs. The whole system is described by

$$
H=H_{\text {leads }}+H_{T}+H_{\text {sys }} .
$$


$H_{\text {leads }}=\sum_{j=1,2} \sum_{\alpha=S, D} \sum_{k} \varepsilon_{k j \alpha} c_{k j \alpha}^{\dagger} c_{k j \alpha}$, is the Hamiltonian of the source (S) and drain (D) leads for transports through the left $(j=1)$ and right $(j=2)$ dots. $H_{T}=\sum_{j=1,2} \sum_{\alpha=S, D} \sum_{k} t_{j \alpha} d_{j}^{\dagger} c_{k j \alpha}+$ H.c. describes the tunneling between the leads and the dots. The last term is the summed system Hamiltonian of the quantum dots and the tunnel-coupled MBSs [17, 19, 23, 26]

$$
\begin{aligned}
H_{\mathrm{sys}} & =\sum_{j=1,2} \epsilon_{j} d_{j}^{\dagger} d_{j}+\frac{i}{2} \epsilon_{M} \gamma_{1} \gamma_{2} \\
& +\lambda_{1}\left(d_{1}^{\dagger}-d_{1}\right) \gamma_{1}+i \lambda_{2}\left(d_{2}^{\dagger}+d_{2}\right) \gamma_{2}
\end{aligned}
$$

In the above Hamiltonians, $d_{j}^{\dagger}\left(d_{j}\right)$ and $c_{j \alpha k}^{\dagger}\left(c_{j \alpha k}\right)$ are, respectively, the electron creation (annihilation) operators of the quantum dots and the attached transport leads, with energies $\epsilon_{j}$ and $\varepsilon_{k j \alpha} . \gamma_{1}$ and $\gamma_{2}$ are the Majorana operators, while $\epsilon_{M}=\Delta e^{-\ell / \xi}$ is the overlap coupling amplitude between the MBSs, with $\ell$ the wire length and $\xi$ the superconducting coherence length.

For the sake of calculation convenience, we move from the Majorana to regular fermion ( $f$ and $f^{\dagger}$ ) representation, via the transformation of $\gamma_{1}=f^{\dagger}+f$ and $\gamma_{2}=i\left(f^{\dagger}-f\right)$. The system Hamiltonian is accordingly rewritten as

$$
\begin{aligned}
H_{\mathrm{sys}}= & \epsilon_{M} f^{\dagger} f+\sum_{j=1,2}\left\{\epsilon_{j} d_{j}^{\dagger} d_{j}\right. \\
& \left.+\lambda_{j}\left[\left(d_{j}^{\dagger}+\eta d_{j}\right) f+\text { H.c }\right]\right\} .
\end{aligned}
$$

Here we introduce a parameter $\eta$ to distinguish the Majorana fermion $(\eta=1)$ from the regular bound state (RBS, $\eta=0$ ). This will be useful for latter comparison. Associated with the above system Hamiltonian, we introduce the state basis as $\left|n_{1} n_{f} n_{2}\right\rangle$, where $n_{1,2, f}=0$ or 1 stand for the occupation number of the QDs and the MBSs. One may notice that the tunneling terms in $H_{\text {sys }}$ only conserve charge modulo $2 e$. This reflects the fact that a pair of electrons can be extracted out from the superconductor and can be also absorbed by the condensate. As a consequence, for the MBSs, the Hilbert space of the central system can be split into two subspaces: $\{|100\rangle,|010\rangle,|001\rangle,|111\rangle\}$ with odd parity; and $\{|011\rangle,|101\rangle,|110\rangle,|000\rangle\}$ with even parity. For the RBS, the Hilbert space is the same but does not need to be split by parity consideration. In the following these basis states will be used to calculate the transport currents and their correlation, based on a master equation approach.

For quantum transport, the leads can be regarded as a general environment, while the central device is the system of interest described by a reduced density matrix $\rho(t)$. Moreover, in weak transmission limit, the Born-Markov approximation applies well, for the setup of Fig. 1 which would result in the following master equation [31]

$$
\dot{\rho}=-i \mathcal{L} \rho+\sum_{j=1,2}\left(\Gamma_{j}^{S} \mathcal{D}\left[d_{j}^{\dagger}\right] \rho+\Gamma_{j}^{D} \mathcal{D}\left[d_{j}\right] \rho\right),
$$

where $\mathcal{L} \rho \equiv\left[H_{\text {sys }}, \rho\right]$ and $\mathcal{D}[A] \rho \equiv A \rho A^{\dagger}-\frac{1}{2}\left\{A^{\dagger} A, \rho\right\}$. In Eq. (4) we employ the tunneling rates $\Gamma_{j}^{S(D)}$ for the $j$ th dot coupling to the source $(S)$ and drain $(D)$ leads, respectively. These rates, more specifically, can be determined by the tunnel-coupling amplitudes and the densityof-states of the leads.

The above Born-Markov master equation is valid in the weak transmission limit. This is also equivalent to a large bias limit, since both correspond to ignoring the level's broadening effect, under the condition that the dot level is far from the Fermi levels of the leads, with at least several $\Gamma$ ( $\Gamma$ stands for the level's broadening). The large bias condition has been examined carefully in Ref. [32], while in [33] an improved scheme is proposed to make the new master equation applicable to arbitrary voltages. Therefore, for the setup under study, Eq. (4) is valid in the large bias regime. Similar equation under the Born-Markov approximation has been applied in the recent work for Majorana systems as well [21, 34]. The large bias condition may raise a concern for its effect on the Majorana bound states. In the context of topological quantum computation, it was analyzed that a tunnel-coupling to electronic reservoir would be detrimental to the Majorana qubit [35]. However, in our present work (as well as in any other Majorana detection scheme), the major concern is not the Majorana qubit, but the Majorana signature in transport currents. In principle, from the general rule of measurement, the backaction (decoherence) is unavoidable. However, it does not destroy the Majorana signature. In either the steady-state current or noise spectrum, the transport backaction only results in a broadening effect on the conductance or noise spectral peak. Finally, it should be noted that a large bias does not necessarily imply a strong transport current, since the coupling between the dot and leads can be very weak.

Based on Eq. (4), the source and drain currents through each dot can be simply calculated by

$$
I_{j}^{S}=e \Gamma_{j}^{S}\left(1-n_{j}\right), \quad I_{j}^{D}=e \Gamma_{j}^{D} n_{j},
$$

where the occupation of the corresponding quantum dot is calculated by $n_{j}=\operatorname{Tr}\left[d_{j}^{\dagger} d_{j} \rho(t)\right]$. In particular, using the stationary density matrix $(\bar{\rho})$, the steady-state currents can be obtained.

The master equation also allows us to calculate, very conveniently, the correlation of currents [31]. In this work, we focus on the cross correlation of currents through the different dots: $G_{i j}\left(t_{2}, t_{1}\right)=\mathrm{E}\left[I_{i}\left(t_{2}\right) I_{j}\left(t_{1}\right)\right]$, where $E[\cdots]$ stands for the ensemble average in the quantum trajectory approach 31]. More specifically, we consider only the cross correlation of the drain currents (denoted here by $I_{j(i)}$ for brevity). The current is defined through $I_{j}(t)=e d N_{j}(t) / d t$ and the average is determined by $\mathrm{E}\left[d N_{j}(t)\right]=d t \operatorname{Tr}\left[d_{j}^{\dagger} d_{j} \rho(t)\right]$. In steady state, the cross correlation is a function of the time difference owing to the time-translational invariance, i.e., $G_{i j}\left(t_{2}, t_{1}\right)=G_{i j}\left(t_{2}-\right.$ $\left.t_{1}\right) \equiv G_{i j}(t)$. Therefore we have

$$
\begin{aligned}
G_{i j}(t)= & e^{2} \operatorname{Tr}\left\{\Gamma_{i}^{D} d_{i}\left[e^{\widetilde{\mathcal{L}} t}\left(\Gamma_{j}^{D} d_{j} \bar{\rho} d_{j}^{\dagger}\right)\right] d_{i}^{\dagger}\right\} \\
& =e^{2} \Gamma_{i}^{D} \Gamma_{j}^{D} \operatorname{Tr}\left[d_{i} \tilde{\rho}_{j}(t) d_{i}^{\dagger}\right] .
\end{aligned}
$$




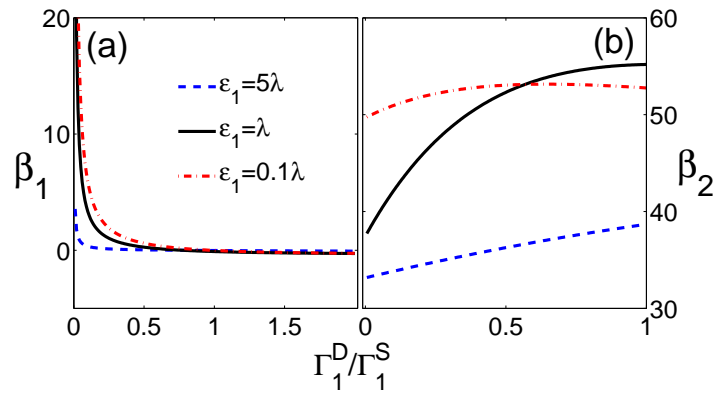

FIG. 2: (Color online) Cross-correlation of the steady state currents, plotted by the scaled difference of the source and drain currents, $\beta_{j}=\left(\bar{I}_{j}^{S}-\bar{I}_{j}^{D}\right) / \bar{I}_{j}^{D}$, with $j=1(2)$ denoting the left (right) dot. The cross-correlation is manifested by modulating $\Gamma_{1}^{D} / \Gamma_{1}^{S}$ and $\epsilon_{1}$ of the left dot, via their effects on $\beta_{2}$ of the right dot. Parameters: $\lambda_{1}=\lambda_{2}=\lambda, \epsilon_{M}=\epsilon_{2}=\lambda, \Gamma_{1}^{S}=\Gamma_{2}^{S}=\lambda$, and $\Gamma_{2}^{D}=0.01 \lambda$.

Here the Liouvillian propagator $e^{\widetilde{\mathcal{L}} t}$ describes the state evolution governed by Eq. (4). From the post-selected state after a jump, i.e., $\tilde{\rho}_{j}(0)=d_{j} \bar{\rho} d_{j}^{\dagger}$, we introduced $\tilde{\rho}_{j}(t) \equiv e^{\widetilde{\mathcal{L}} t} \tilde{\rho}_{j}(0)$ which obviously satisfies Eq. (4).

The spectral density of cross correlation is given by the Fourier transform of $G_{i j}(t)$, which yields

$$
S_{i j}(\omega)=e^{2} \Gamma_{i}^{D} \Gamma_{j}^{D} \operatorname{Tr}\left[d_{i} \tilde{\rho}_{j}(\omega) d_{i}^{\dagger}+d_{j} \tilde{\rho}_{i}(-\omega) d_{j}^{\dagger}\right] .
$$

In deriving this result, we have used the property $G_{21}(t)=G_{12}(-t)$ and introduced the definition $\tilde{\rho}_{j}( \pm \omega)=\int_{0}^{\infty} \tilde{\rho}_{j}(t) e^{ \pm i \omega t} d t$. Straightforwardly, via the technique of Laplace transformation, $\tilde{\rho}_{j}(\omega)$ can be solved from Eq. (4). Moreover, we would like to employ a symmetrized spectral density, $S(\omega)=\left[S_{21}(\omega)+S_{12}(\omega)\right] / 2$, to characterize the cross correlation. We have

$$
S(\omega)=e^{2} \Gamma_{1}^{D} \Gamma_{2}^{D} \operatorname{Re}\left\{\operatorname{Tr}\left[d_{1} \tilde{\rho}_{2}(\omega) d_{1}^{\dagger}+d_{2} \tilde{\rho}_{1}(\omega) d_{2}^{\dagger}\right]\right\} .
$$

Also, we will use the Fano factor to describe the zerofrequency correlation. The Fano factor is defined as $F=$ $S(0) / 2 e \sqrt{\bar{I}_{1} \bar{I}_{2}}$, where $\bar{I}_{1(2)}=\left[\bar{I}_{1(2)}^{S}+\bar{I}_{1(2)}^{D}\right] / 2$ is the average stationary current through the left (right) dot.

In Refs. 26, 27], the isolated dot-MBSs-dot system was studied. Remarkably, it was found that, even in the limit $\epsilon_{M}=0$, a novel process of teleportationlike electron transfer between the distant dots is possible, due to the inherent nonlocal nature of the Majorana fermion. If $\epsilon_{M} \neq 0$, it also leads to a discontinuity of the tunneling conductance through one of the dots, under the MBSmediated influence of the other dot. In the following, we will show: (i) In the limit $\epsilon_{M}=0$, there is no crosscorrelation between the transport currents through the two individual dots. This is an interesting result, by noting the presence of electron transfer between the dots, mediated by the MBSs. (ii) For $\epsilon_{M} \neq 0$, distinct Majorana signatures can appear in the cross correlation of currents.

Stationary and Transient Correlation.- Let us consider first the steady-state currents through the two dots

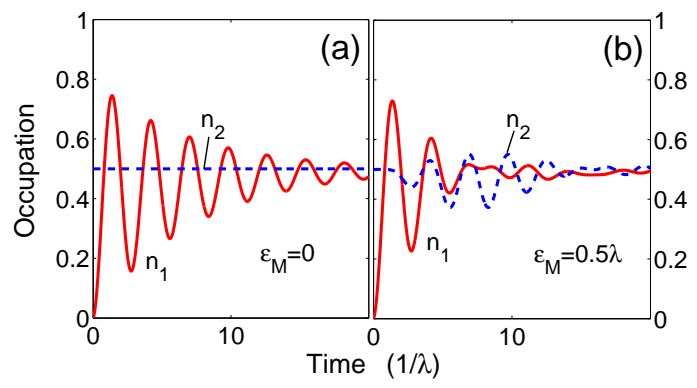

FIG. 3: (Color online) Transient dynamics of the right dot in response to an electron jump from the left dot to its drain lead, based on the steady state. The occupation of the $j$ th dot is obtained by $n_{j}(t)=\operatorname{Tr}\left[d_{j}^{\dagger} d_{j} \rho(t)\right]$, with $\rho(t)$ evolved from the specific "initial" state $\rho(0)=d_{1} \bar{\rho} d_{1}^{\dagger} / \operatorname{Tr}\left(d_{1} \bar{\rho} d_{1}^{\dagger}\right)$. Assuming $\lambda_{1}=\lambda_{2}=\lambda$, we give the result in (a) for $\epsilon_{M}=0$ and in (b) for $\epsilon_{M}=0.5 \lambda$. Other parameters: $\epsilon_{1}=\epsilon_{2}=\lambda$, and $\Gamma_{S(D)}^{1(2)}=0.1 \lambda$.

and their correlation mediated by the MBSs. We notice that, owing to coupling to the MBS, in the transport through a quantum dot the source current is in general not equal to the drain current [20]. Their difference, in essence, is compensated by a current flowing through the nanowire into or from the superconductor condensate. For our present double-dot system, owing to the similar reason, we introduce $\beta_{j}=\left(\bar{I}_{j}^{S}-\bar{I}_{j}^{D}\right) / \bar{I}_{j}^{D}(j=1,2)$ to qualify this unique feature. In Fig. 2, we use this factor to illustrate the cross correlation. Specifically, we alter $\Gamma_{1}^{D} / \Gamma_{1}^{S}$, and examine its effect on $\beta_{1}$ and in particular on $\beta_{2}$. The results in Fig. 2 correspond to $\epsilon_{M} \neq 0$, where the existence of cross correlation is obviously indicated, while its peculiar feature is to be further exploited in the following. We would like to mention that, if $\epsilon_{M}=0$, altering $\Gamma_{1}^{D} / \Gamma_{1}^{S}$ would have no effect on $\beta_{2}$. This result completely differs from what happens for two quantum dots coupled through a regular bound state.

To further illustrate the MBS-mediated cross correlation, we now turn to the transient dynamics. To be specific, we consider how a disturbance on the left dot would affect the transport through the right one. Based on the steady state, the disturbance can be introduced by a tunneling event of an electron from the left dot to its drain lead. This consideration defines a particular "initial" state, $d_{1} \bar{\rho} d_{1}^{\dagger} / \operatorname{Tr}\left(d_{1} \bar{\rho} d_{1}^{\dagger}\right)$. Then, we consider the transient currents associated with this new state.

First, in Fig. 3(a) we show the result in the limit of $\epsilon_{M}=0$. We find that the right current is unaffected by the disturbance on the left dot, which is in agreement with the above tunnel-rate modulation. This result straightforwardly indicates a zero cross-correlation between the currents through the two dots. This behavior is indeed not obvious at all, by two observations: (i) If we consider the two dots being coupled by a regular bound state, even for nonaligned levels of $\epsilon_{1}=\epsilon_{2}=\lambda$ and $\epsilon_{M}=0$, we find that the cross-correlation between $I_{1}$ and $I_{2}$ is nonzero. (ii) It was found in Refs. [26, 27], where the isolated dot-MBSsdot system was analyzed, that the state $|100\rangle$ can evolve 

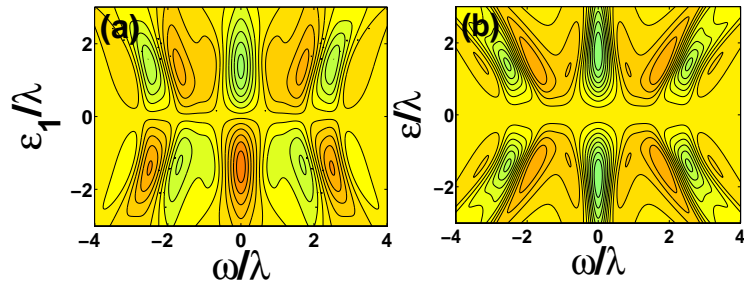

S
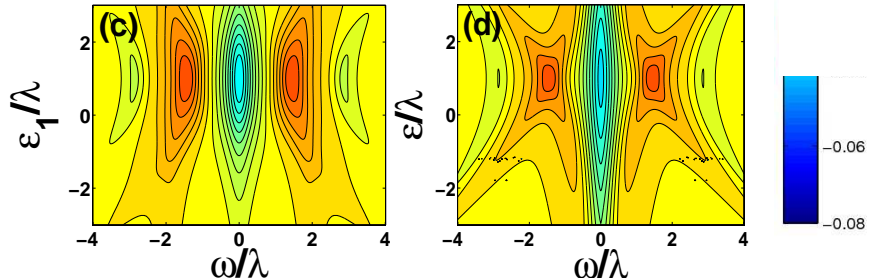

FIG. 4: (Color online) Spectral density of the cross correlation versus two types of dot-level modulations: in (a) and (c) modulation of the left dot level $\left(\epsilon_{1}\right)$ only; and in (b) and (d) simultaneous modulation of the both dot levels $\left(\epsilon_{1}=\epsilon_{2}=\epsilon\right)$. For the single dot $\epsilon_{1}$-modulation, we assume $\epsilon_{2}=\epsilon_{M}=\lambda$, where $\lambda$ is the common coupling strength between the QD and the MBS (i.e., $\lambda_{1}=\lambda_{2}=\lambda$ ). In (a) and (b) we show the results for the MBSs with $\epsilon_{M}=\lambda$, while the results in (c) and (d) correspond to a regular bound state (RBS) with energy $\epsilon_{M}$, as a comparison. In all these results, we have set $\Gamma_{1(2)}^{S(D)}=0.3 \lambda$.

to $|001\rangle$ in short timescale even in the limit $\epsilon_{M}=0$. This seemingly indicates that the right current $I_{2}$ should be affected by the left-dot disturbance in this limit.

In Fig. 3(b) we show the result of $\epsilon_{M} \neq 0$, assuming $\epsilon_{M}=0.5 \lambda$ and $\epsilon_{1}=\epsilon_{2}=\lambda$. In the transformed basis, this setup does not look too much different from $\epsilon_{M}=0$. However, we find in Fig. 3(b) that the current through the right dot is considerably affected by the disturbance on the left dot. Moreover, as to be shown in the following, its correlation behavior differs significantly from that mediated by a regular bound state with this same energy $\left(\epsilon_{M}\right)$.

Cross Correlation Spectrum.- Below we employ the spectral density function Eq. (8) to analyze the transient dynamics. In Fig. 4 we present a contour-plot for the spectral density of the cross correlation, as a function of frequency and dot-level modulations. In Fig. 4(a) and (b) we show the results for MBSs and in (c) and (d) for RBS, using the same energy $\left(\epsilon_{M} \neq 0\right)$ for the sake of comparison. We find all the results in Fig. 4(a)-(d) an even function of the frequency. This is because of the property $G_{i j}(t)=G_{j i}(-t)$ and the symmetrization procedure for the cross-correlation function which leads to Eq. (8). More interesting is that, for the MBS-mediated correlation, the spectral density function would have different dependence on the dot-level modulations. That is, for the level modulation of single dot, the spectral density in Fig. 4(a) is antisymmetric with respect to the energy of the Majorana zero mode. In contrast, however, the spectrum in Fig. 4(b) is symmetric under the simultaneous modulation of the double-dot energy levels. We conclude that this is one of the important signatures resulted from the

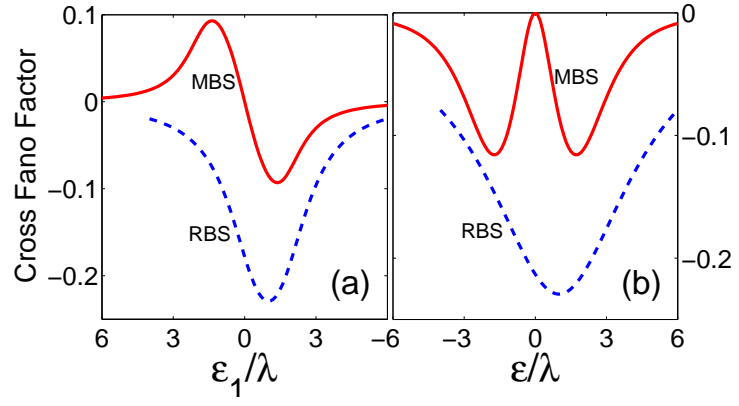

FIG. 5: (Color online) Fano factor of the cross correlation, which corresponds to the zero-frequency noise and is defined in the main text. All the parameters used in this figure are the same as in Fig. 4.

Majorana nonlocal correlation. In Fig. 4(c) and (d) we observe that the cross-correlation mediated by a regular bound state, differently, are always symmetric under both sorts of dot-level modulations.

To show more clearly the symmetry behavior mentioned above, we plot in Fig. 5 the zero-frequency Fano factor. We find that the results for MBSs, being either symmetric or antisymmetric, are centered at the Majorana zero mode and have a vanished cross Fano factor there. This result cannot be interpreted by the usual picture of resonant transfer in terms of matching the energy levels. Actually, in the transformed representation, the two MBSs are "combined" to form a regular fermion with energy $\epsilon_{M}$. This is likely to indicate that the cross correlation, including the frequency-dependent spectrum in Fig. 4(a) and (b), should be centered at the "effective" energy $\epsilon_{M}$. Moreover, when one of the dot levels is in resonance with the Majorana zero mode, the vanished cross correlation is far beyond our intuition. As a sharp comparison, in Fig. 5 we display the results from the RBS-mediated correlation. Evidently, we find that the cross Fano factor is always symmetric with respect to the RBS energy, for the both types of dot-level modulations. Moreover, at the symmetry center the cross correlation is nonzero. Therefore, these remarkable differences, together also with the entire "lineshape" in response to the dot-level modulations, provide us very useful information to identify the emerged Majorana bound states.

We became aware of a couple of recent studies on the Majorana-mediated cross correlation. In Ref. [36] the motion (transport) of Majorana fermion through a Hanbury Brown-Twiss type interferometer is considered. In this four terminal transport setup, it is found that the cross correlation of currents reveals a novel "+(-)" sign signature and an absence of partition noise after adding a quantumpoint-contact, both of which can be traced back to the Majorana nature of the carriers. Being more relevant to the present work, in Refs. [21, 22], the series transport through two quantum dots coupled by a pair of MBSs is analyzed, where the analogous suppressed cross correlation is observed as well, under the same condition as mentioned above. Therefore, the vanished cross correlation found in both the parallel and series transport setups 
should originate from the same Majorana physics, being of interest and worth further clarification.

Experimental Feasibility.- For possible experimental demonstration, the semiconductor nanowire can be the $\mathrm{InSb}$ wire, as utilized in the recent experiment [9]. The InSb nanowire has a large $g$-factor $(g \simeq 50)$, and a strong Rashba spin-orbit interaction (with energy $\sim 50 \mu \mathrm{eV}$ ). For magnetic field of 0.15 Tesla, the Zeeman splitting $\left(V_{z} \simeq\right.$ $225 \mu \mathrm{eV}$ ) starts to exceed the induced superconducting gap $\Delta \simeq 200 \mu \mathrm{eV}$. Moreover, a low temperature such as $T=100 \mathrm{mK}$ can suppress the thermal excitation of the Majorana zero mode to higher energy states. Under these conditions, the MBSs may appear at the ends of the nanowire [9]. In practice, the energy level modulation of the quantum dot can be implemented by gate voltage. Utilizing the gate-voltage technique, one can also control the tunneling rates $\Gamma_{S(D)}^{1(2)}$ and the coupling energy $\lambda$ to the order of a few $\mu \mathrm{eV}$, which can maintain electron oscillations between the QD and MBSs within a coherence time longer than nanoseconds. This magnitude order of coupling values also allows the condition $k_{B} T<e V<E_{z}$ to define a broad range of bias voltage across the quan- tum dot, satisfying the large bias condition to guarantee the Born-Markov master equation.

Summary.- We analyzed the cross correlation mediated by a pair of Majorana bound states, based on a specific setup of parallel transport through two quantum dots. The essential "dot-MBSs-dot" building block of the setup is experimentally accessible using the nowaday state-ofthe-art technology, particularly based on a semiconductor nanowire realization. From the cross correlation of the parallel currents, we revealed several unusual Majorana signatures, including the symmetry and antisymmetry behaviors of the spectral density in response to the dot-level modulations, and the vanished cross correlation when any of the dot-levels is in resonance with the Majorana zero mode. These signatures are of interest and beyond possible interpretations based on the conventional regular fermion system.

Acknowledgments. - This work was supported by the Major State Basic Research Project (Nos. 2011CB808502 \& 2012CB932704), and the NNSF of China (No. 101202101).
[1] E. Majorana, Nuovo Cimento 14, 171 (1937).

[2] F. Wilczek, Nat. Phys. 5, 614 (2009); M. Franz, Physics 3, 24 (2010).

[3] G. Moore and N. Read, Nuclear Physics B 360, 362(1991).

[4] A. Y. Kitaev, Physics-Uspekhi 44, 131 (2001).

[5] N. Read and D. Green, Phys. Rev. B 61, 10267 (2000).

[6] Y. Oreg, G. Refael, and F. von Oppen, Phys. Rev. Lett. 105, 177002 (2010).

[7] J. D. Sau, R. M. Lutchyn, S. Tewari, and S. Das Sarma, Phys. Rev. Lett. 104, 040502 (2010).

[8] J. D. Sau, S. Tewari, and S. Das Sarma, Phys. Rev. B 85, 064512 (2012).

[9] V. Mourik, K. Zuo, S. M. Frolov, S. R. Plissard, E. P. A. M. Bakkers, L. P. Kouwenhoven, Science, 336, 1003 (2012).

[10] M. T. Deng, C. L. Yu, G. Y. Huang, M. Larsson, P. Caroff, H. Q. Xu, arXiv:1204.4130.

[11] L. P. Rokhinson, X. Liu, J. K. Furdyna, arXiv:1204.4212.

[12] A. Das, Y. Ronen, Y. Most, Y. Oreg, M. Heiblum, and H. Shtrikman, arXiv:1205.7073.

[13] A. Y. Kitaev, Ann. Phys. (N.Y.) 303, 2 (2003).

[14] A. Stern, Nature (London) 464, 187 (2010).

[15] C. Nayak, S. H. Simon, A. Stern, M. Freedman, and S. Das Sarma, Rev. Mod. Phys. 80, 1083 (2008).

[16] J. Alicea, Y. Oreg, G. Refael, F. von Oppen, and M. P. A. Fisher, Nature Physics 7, 412 (2011).

[17] D. E. Liu and H. U. Baranger, Phys. Rev. B 84,201308 (2011).

[18] C.J. Bolech and E. Demler, Phys. Rev. Lett. 98, 237002 (2007).

[19] K. T. Law, P. A. Lee, and T. K. Ng, Phys. Rev. Lett. 103, 237001 (2009).
[20] Y. S. Cao, P. Y. Wang, G. Xiong, M. Gong, and X. Q. Li, Phys. Rev. B 86, 115311 (2012).

[21] H. F. Lü, H. Z. Lu, and S. Q. Shen, Phys. Rev. B 86, 075318 (2012).

[22] B. Zocher and B. Rosenow, arXiv:1208.4092

[23] R. M. Lutchyn, J. D. Sau, and S. Das Sarma, Phys. Rev. Lett. 105, 077001 (2010).

[24] L. Fu and C. L. Kane, Phys. Rev. B 79, 161408 (2009).

[25] J. Nilsson, A. R. Akhmerov, and C. W. J. Beenakker, Phys. Rev. Lett. 101,120403 (2008).

[26] S. Tewari, C. Zhang, S. Das Sarma, C. Nayak, and D. H. Lee, Phys. Rev. Lett. 100, 027001 (2008).

[27] P. Y. Wang, Y. S. Cao, M. Gong, S. S. Li, and X. Q. Li, arXiv: 1208.3738

[28] O. Sauret, D. Feinberg, and T. Martin, Phys. Rev. B 69, 035332 (2004).

[29] C. H. Bennett, G. Brassard, C. Crepeau, R. Jozsa, A. Peres, and W.K. Wootters, Phys. Rev. Lett. 70, 1895(1993).

[30] L. Fu, Phys. Rev. Lett. 104, 056402 (2010).

[31] H. B. Sun and G. J. Milburn, Phys. Rev. B 59 , 10748 (1999).

[32] F. Li, X. Q. Li, W. M. Zhang and S. Gurvitz, Europhys. Lett. 88, 37001 (2009).

[33] J. Li, J. Jin, X. Q. Li, and Y. J. Yan, arXiv:1110.4417.

[34] M. Leijnse and K. Flensberg, Phys. Rev. B 84, 140501(R) (2011).

[35] J. C. Budich, S. Walter, and B. Trauzettel, Phys. Rev. B 85, 121405(R) (2012).

[36] G. Strübi, W. Belzig, M. S. Choi, and C. Bruder, Phys. Rev. Lett. 107, 136403 (2011). 